\title{
Database of polyacetylene-containing foods for estimation of population intake
}

\author{
Sarah Warner ${ }^{1}$, Chris Seal ${ }^{1}$, Sumanto Haldar ${ }^{2}$ and Kirsten Brandt ${ }^{1}$ \\ ${ }^{1}$ Newcastle University, Newcastle upon Tyne, NE1 $7 R U$ and ${ }^{2}$ Singapore Institute for Clinical Sciences, Brenner Centre \\ for Molecular Medicine, 30 Medical Drive, Singapore
}

A group of compounds called polyacetylenes (including falcarinol, falcarindiol and falcarindiol-3-acetate), naturally present in carrots and other vegetables of the Apiaceae family, is gaining interest due to their potential anti-inflammatory ${ }^{(1)}$ and anti-cancer effects ${ }^{(2)}$. Unlike the well investigated carotenoids also present in carrots, the population intake of polyacetylenes is not well known, so polyacetylene contents in different foods were analysed to provide a database suitable for combining with diet data in order to estimate intake of these compounds.

Data from the Newcastle $85+$ study $^{(3)}$ was used to compile a list of all commonly eaten polyacetylene-rich vegetables and mixed dishes that contain them. For each food type, meals or raw materials were procured from different sources, resulting in a total of 96 independent samples. Home-made meals were prepared according to McCance and Widdowson's 'The Composition of Foods ${ }^{\text {(4) }}$ or recipes found on UK based websites. The foods were freeze dried, extracted by ethyl acetate and analysed by HPLC. The foods were grouped into 8 categories as shown in Figure 1.

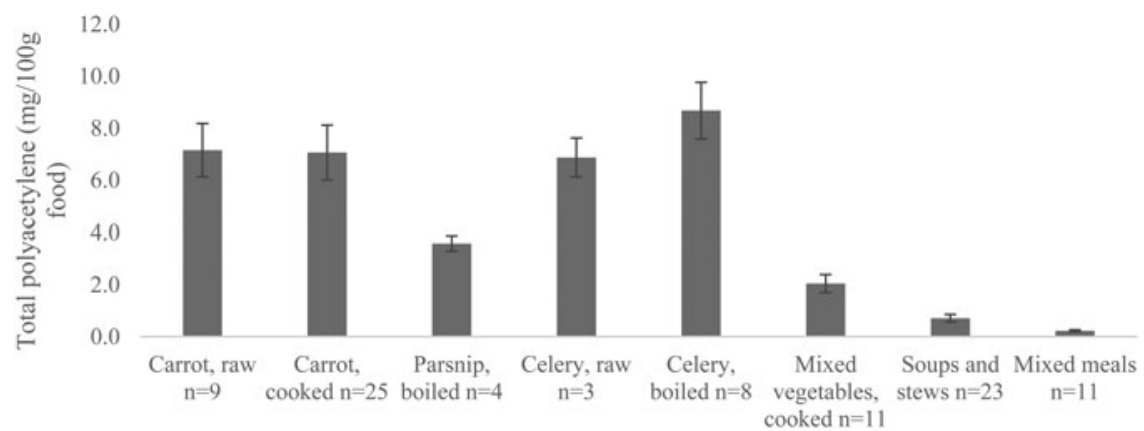

Fig. 1. Overview of polyacetylene content in relevant food groups. Data are sum of falcarinol, falcarindiol and falcarindiol-3-acetate. Error bars show the standard error of the mean.

Cooked vegetables tend to have higher polyacetylene concentrations than raw, due to loss of water and sugar during cooking. Mixed vegetables as well as soups, stews and mixed meals are a mixture of polyacetylene-rich vegetables and other foods so have lower average polyacetylene concentrations. The results of this study can be combined with dietary data about portion sizes and number of portions consumed, to obtain estimates of total polyacetylene intake in a population or an individual participating in a study.

The database is available for all relevant research projects and contains details of the food types analysed (with McCance and Widdowson food codes), with the polyacetylene data specified for each of the 3 analysed compounds. Please contact s.r.warner@newcastle.ac.uk if you are interested in using this data.

This work was supported by a studentship from the Agriculture and Horticulture Development Board, UK.

1. Metzger BT, Barnes DM, Reed JD (2008) J Agr Food Chem 56, 3554-60.

2. Zaini RG, Brandt K, Claench MR, Le Maitre CL (2012) Anti-Cancer Agent Me 12, 640-52.

3. Collerton J, Barrass K, Bond J, Eccles M, Jagger C, James O, et al. (2007) BMC Geriatr 7, 14

4. McCance RA, Widdowson EM (2002) The Composition of Foods. 6 ed. Royal Society of Chemistry. 